CUBO A Mathematical Journal

Vol.20, $N^{\underline{Q} 02,(01-12) .}$ June 2018

\title{
An approach to F. Riesz representation Theorem
}

\author{
Rafael del Rio, Asaf L. Franco and Jose A. Lara \\ Departamento de Física Matemática, \\ Instituto de Investigaciones en Matemáticas Aplicadas y en Sistemas, \\ Universidad Nacional Autónoma de México \\ C.P. 04510, CDMX, México. \\ delrio@iimas.unam.mx, asaflevif@hotmail.com, nekrotzar.ligeti@gmail.com
}

\begin{abstract}
In this note we give a direct proof of the F. Riesz representation theorem which characterizes the linear functionals acting on the vector space of continuous functions defined on a set K. Our start point is the original formulation of Riesz where $\mathrm{K}$ is a closed interval. Using elementary measure theory, we give a proof for the case $\mathrm{K}$ is an arbitrary compact set of real numbers. Our proof avoids complicated arguments commonly used in the description of such functionals.
\end{abstract}

\begin{abstract}
RESUMEN
En esta nota, damos una demostración directa del teorema de representación de F. Riesz que caracteriza los funcionales lineales actuando en el espacio vectorial de funciones continuas definidas en un conjunto K. Nuestro punto de partida es la formulación original de Riesz, donde K es un intervalo cerrado. Usando teoría elemental de la medida, damos una demostración para el caso en que $\mathrm{K}$ es un conjunto arbitrario compacto de números reales. Nuestra demostración evita argumentos complicados comúnmente usados en la descripción de dichos funcionales.
\end{abstract}

Keywords and Phrases: Riesz representation theorem, positive linear functionals, RiemannStieltjes integral.

2010 AMS Mathematics Subject Classification: 46E11,46E15,28A25. 


\section{Introduction}

The Riesz representation theorem is a remarkable result which describes the continuous linear functionals acting on the space of continuous functions defined on a set $\mathrm{K}$. It is very surprising that all these functionals are just integrals and vice versa. In case $\mathrm{K}$ is a closed interval of real numbers, any such functional is represented by Riemann-Stieltjes integral, which is a generalization of the usual Riemann integral. This was first announced by F. Riesz in 1909 [14. In case K is compact set (not necessarily a closed interval), then a more general concept of integral is needed, because the Riemann-Stieltjes integral used by Riesz is defined only for functions on intervals. In this work, we prove that there is a short path between the two cases.

Besides its aesthetic appeal, the above mentioned theorem has far-reaching applications. It allows a short proof of the Kolmogoroff consistency theorem, see [3] thm 10.6.2., and can be used to give an elegant proof of the spectral theorem for selfadjoint bounded operators, see section VII.2 of [13. Both these theorems are main results in probability and functional analysis respectively. Moreover, the entire theory of integration for general spaces can be recovered using the theorem of Riesz. See for example [19], where the Lebesgue measure on $\mathbb{R}^{n}$ is constructed. More generally it can also be used to show the existence of the Haar measure on a group, see [3] chap. 9.

In this note we give a short proof of the Riesz representation theorem for the case $\mathrm{K}$ is an arbitrary compact set of real numbers, see Theorem 3.1 below. This is interesting because in many situations we have a compact set which is not a closed interval. To prove the spectral theorem, for example, one considers the set of continuous functions defined on the spectrum of selfadjoint bounded operator, which is a compact set of $\mathbb{R}$, but not necessarily a closed interval. We get our result starting from the nondecreasing function that appears in the Riemann-Stieltjes integral representation of Riesz original formulation. To this function we associate a measure which is used to integrate over general compact sets. Then we show how this Lebesgue integral representation can be seen as a Riemann-Stieltjes integral. Our proof is new, avoids technical arguments which appear frequently in proofs of Riesz theorem, it is elementary, direct and quite simple.

\section{Preliminaries}

Let us introduce first some definitions and notations we shall use.

\subsection{Definitions and notation.}

Let $\mathcal{C}(\mathrm{K}):=\{\mathrm{f}: \mathrm{K} \rightarrow \mathbb{R}: \mathrm{f}$ continuous $\}$ where $\mathrm{K}$ is a compact subset of $\mathbb{R}$, the real numbers. A functional is an assignment $L: \mathcal{C}(K) \rightarrow \mathbb{R}$. The functional is linear if $L\left(c_{1} f+c_{2} g\right)=c_{1} L(f)+c_{2} L(g)$ for all $f, g \in \mathcal{C}(K), c_{1}, c_{2} \in \mathbb{R}$. It is continuous if there exists a fixed $M>0$ such that $|L f| \leq M\|f\|_{\infty}$ 
for all $f \in \mathcal{C}(K)$, where $\|\cdot\|_{\infty}$ denotes the uniform norm, that is, $\|f\|_{\infty}=\sup \{|f(x)|: x \in K\}$. We define the norm of such functional as

$$
\|\mathrm{L}\|_{\mathcal{C}(\mathrm{K})}=\|\mathrm{L}\|=\sup \left\{|\mathrm{L}(\mathrm{f})|: \mathrm{f} \in \mathcal{C}(\mathrm{K}) \text { and }\|\mathrm{f}\|_{\infty} \leq 1\right\},
$$

We denote the set of the linear continuous functionals on $\mathcal{C}(\mathrm{K})$ by $\mathcal{C}(\mathrm{K})^{*}$. It is called the dual space. In general, the dual of normed linear space $X$ is denoted by $X^{*}$. A functional $L$ on $\mathcal{C}(\mathrm{K})$ is said to be a positive if $L(f) \geq 0$ whenever $f(x) \geq 0$ for every $x \in \mathbb{R}$. We use the notation $\mathcal{C}(K)_{+}^{*}$ for the set of positive linear functionals on $\mathcal{C}(\mathrm{K})$.

The function $\alpha:[a, b] \longrightarrow \mathbb{R}$ is said to be normalized, if $\alpha(a)=0$ and $\alpha(t)=\alpha(t+)$, $a<t<b$, that is, $\alpha$ is continuous from the right inside the interval (not at a! If it were right continuous at $a$, theorem (2.1) would not hold for the functional $L(f)=f(a))$. The total variation of a monotone increasing function $\alpha$ is defined as $V(\alpha)=\alpha(b)-\alpha(a)$. We denote the characteristic function of a set $A \subset[a, b]$ by $\mathbf{1}_{A}$ where $\mathbf{1}_{A}(x)=1$ if $x \in A$ and 0 if $x \in[a, b] \backslash A$.

\subsection{Representation theorem for functionals on $\mathcal{C}[a, b]$.}

We formulate the above-mentioned result by F. Riesz as follows:

Theorem 2.1. Let $\mathrm{L}: \mathcal{C}[\mathrm{a}, \mathrm{b}] \longrightarrow \mathbb{R}$ be a positive linear functional. There exists a unique normalized monotone function $\alpha:[\mathrm{a}, \mathrm{b}] \longrightarrow \mathbb{R}$ such that

$$
\mathrm{Lf}=\int_{a}^{b} f(x) d \alpha(x) .
$$

The integral is understood in the sense of Riemann-Stieltjes. Moreover $\|\mathrm{L}\|=\mathrm{V}(\alpha)$.

The Riemann-Stieltjes integral is a generalization of the Riemann integral, where instead of taking the length of the intervals, a $\alpha$-weighted length is taken. For an interval I the $\alpha$-length is given by $\alpha(\mathrm{I})=\alpha(\mathrm{y})-\alpha(\mathrm{x})$, where $x, y$ are the end points of I and $\alpha$ is a function of finite variation. The integral of a continuous function $f$ on $[a, b]$ is defined as the limit, when it exists, of the sum $\sum_{i} f\left(c_{i}\right) \alpha\left(I_{i}\right)$ where $\left\{I_{i}\right\}$ is a finite collection of subintervals whose endpoints form a partition of $[a, b]$ and $c_{i} \in I_{i}$. See [17] p.122.

There are different proofs of the above theorem, see for example [22]. Here we will give a sketch of the proof which uses the following result about extensions of functionals known as the Hahn-Banach theorem:

Let $\mathrm{X}$ a normed linear space, $\mathrm{Y}$ a subspace of $\mathrm{X}$, and $\lambda$ an element of $\mathrm{Y}^{*}$. Then there exists a $\Lambda \in X^{*}$ extending $\lambda$ with the same norm. See [13] for a proof.

Proof of theorem [2.1]. We may assume that $[a, b]=[0,1]$. Since $L \in \mathcal{C}[0,1]^{*}$ we use Hahn-Banach theorem to conclude the existence of $\Lambda \in \mathrm{B}[0,1]^{*}$ such that $\|\Lambda\|=\|\mathrm{L}\|$ and $\mathrm{L}=\Lambda$ on $\mathcal{C}[0,1]$ and 
where $B[0,1]$ is the set of bounded functions on $[0,1]$.

Let us define the functions $\mathbf{1}_{x}:=\mathbf{1}_{[0, x]}$, that is $\mathbf{1}_{x}(t)=1$ when $t \in[0, x]$ and zero otherwise. Set $\alpha(x)=\Lambda\left(\mathbf{1}_{x}\right)$ for all $x \in[0,1]$.

Now for $f \in \mathcal{C}[0,1]$, define

$$
f_{n}=\sum_{j=1}^{n} f(j / n)\left(\mathbf{1}_{j / n}-\mathbf{1}_{(j-1) / n}\right) .
$$

Since $f$ is continuous, it is uniformly continuous on $[0,1]$ and so $\left\|f_{n}-f\right\|_{\infty} \rightarrow 0$. Thus

$$
\lim _{n} \Lambda\left(f_{n}\right)=\Lambda(f)=L(f) .
$$

Using the definition of $\alpha$ we get

$$
\Lambda\left(f_{n}\right)=\sum_{j=1}^{n} f(j / n)(\alpha(j / n)-\alpha((j-1) / n)) .
$$

This in turn implies

$$
\Lambda(f)=\lim _{n} \Lambda\left(f_{n}\right)=\int_{0}^{1} f d \alpha
$$

Now to see that $\|\mathrm{L}\|=\mathrm{V}(\alpha)$ :

Let $\varepsilon>0$ and choose $f \in \mathcal{C}[0,1]$ such that $\|f\|_{\infty} \leq 1$ and $\|\mathrm{L}\| \leq|\mathrm{L}(\mathrm{f})|+\varepsilon$, we apply (2.1) and we get

$$
\|\mathrm{L}\| \leq|\mathrm{L}(\mathrm{f})|+\varepsilon=\left|\int_{0}^{1} f(x) \mathrm{d} \alpha(x)\right|+\varepsilon \leq \alpha(1)-\alpha(0)+\varepsilon=\mathrm{V}(\alpha)+\varepsilon .
$$

It is possible to normalize $\alpha$ and in this case we easily have the other inequality, that is,

$$
\mathrm{V}(\alpha)=\alpha(1)-\alpha(0)=\alpha(1)=\Lambda\left(1_{1}\right) \leq\|\Lambda\|=\|\mathrm{L}\|
$$

\section{Remarks.}

(1) The standard textbook's proof uses Hahn-Banach's theorem ([10],[22]), but the original proof of F. Riesz does not use it. See [17] section 50 and [15, [16]. 
(2) E. Helly [8] should have similar results. J. Radon extended theorem 2.1 to compact subsets $K \subset \mathbb{R}^{n}[12$. S. Banach and S. Saks extended the result to compact metric spaces, see appendix of [21] and [20]. The proof by S. Saks is particularly elegant and clean. For compact Hausdorff spaces the theorem was proven by S. Kakutani 9 and for normal spaces by A. Markoff [11. Nowadays this theorem is also known as Riesz-Markoff or Riesz-MarkoffKakutani theorem. There is a great variety of proofs of F. Riesz theorem using different methods and even category theory see [7. Our proof only uses basic knowledge of measure theory. More information on the history of this theorem can be found in [5] p. 231, the references therein, [23] p.238 and [6].

(3) Positivity of a linear functional L implies continuity of L. To see it, we take the function $\mathbf{1}(x)=1$ for all $x \in K$, then $\mathbf{1} \in \mathcal{C}(K)$ and $|f(x)| \leq\|f\|_{\infty} \mathbf{1}(x)$, therefore

$$
\|f\|_{\infty} \mathbf{1}(x) \pm f(x) \geq 0 \quad \text { implies } \quad\|f\|_{\infty} L(\mathbf{1}) \pm L(f) \geq 0
$$

so $|\mathrm{L}(\mathbf{f})| \leq \mathrm{L}(\mathbf{1})\|\mathrm{f}\|_{\infty}$. See [5] Prop. 7.1.

\section{$3 \quad$ Main Result}

Next theorem is our main result. It is a generalization of Theorem 2.1 to continuous functions defined on arbitrary compact sets $K \subset \mathbb{R}$. Since an ordinary Riemann-Stieltjes integral is not defined for functions on general compact $K$, we shall introduce the Lebesgue integral which makes sense for such functions. In the Appendix, we collect the basic facts and definitions of measure theory we need.

Theorem 3.1. Let $\mathrm{K}$ a compact subset of $\mathbb{R}$ and let $\ell: \mathcal{C}(\mathrm{K}) \rightarrow \mathbb{R}$ be a positive linear functional. Then, there is a unique finite Borel measure $\mu$ such that $\mu(\mathrm{K})=\|\ell\|_{\mathcal{C}(\mathrm{K}) *}$ and

$$
\ell f=\int_{K} f d \mu .
$$

Proof. The proof proceeds in stages.

i) Integral representation. Let $[\mathrm{a}, \mathrm{b}]$ be a closed and bounded interval containing $\mathrm{K}$. Note that the technique used in what follows is independent of this interval. Let $r: \mathcal{C}[\mathrm{a}, \mathrm{b}] \longrightarrow \mathcal{C}(\mathrm{K})$ be the restriction operator, that is, for every $f \in \mathcal{C}[a, b], r(f)(x)=f(x)$ for $x \in K$. It is clear that $r$ is a bounded linear operator, so we can define its transpose operator, see [23] p.11, also known as adjoint, see [22. Recall $r^{t}$ is defined as follows $r^{t}: \mathcal{C}(K)^{*} \rightarrow \mathcal{C}[a, b]^{*}$, $r^{t}(\ell)(f)=\ell(r(f))$ for $f \in \mathcal{C}[a, b]$; the expression $\ell(r(f))$ assigns a scalar to each function $f \in \mathcal{C}[a, b]$. 
Let $\ell$ be a positive linear functional in $\mathcal{C}(K)$ and we define $L f=r^{t}(\ell)(f)=\ell(r f)$. Since $\ell$ and $r$ are positive linear functionals, so is $\mathrm{L}$ and we can apply theorem 2.1] and (c) in the Appendix to find a monotone increasing function $\alpha$ and an associated Borel measure $\mu$ such that

$$
L f=r^{t}(\ell)(f)=\int_{a}^{b} f d \alpha=\int_{a}^{b} f d \mu
$$

for every $f \in \mathcal{C}[a, b]$.

Denote $K^{c}:=[a, b] \backslash K$. We will show that $\mu\left(K^{c}\right)=0$. Let $\varepsilon>0$ and choose $F_{\varepsilon}$ as a closed subset of $\mathrm{K}^{\mathrm{c}}$ such that

$$
\mu\left(K^{c} \backslash F_{\varepsilon}\right)<\varepsilon,
$$

see (a) in the Appendix.

Let $\tilde{f} \in \mathcal{C}[a, b]$ be a continuous function such that $\tilde{f}(x)=1$ if $x \in K, \tilde{f}(x)=0$ if $x \in F_{\varepsilon}$ and $\|\tilde{f}\|_{\infty} \leq 1$. One can take for instance

$$
\tilde{f}(x)=\frac{d\left(x, F_{\varepsilon}\right)}{d\left(x, F_{\varepsilon}\right)+d(x, K)},
$$

where $d(x, A)=\inf _{y \in A}|x-y|$. Note that since $|d(x, A)-d(y, A)| \leq|x-y|$ the function $\mathrm{d}(x, A)$ is even uniformly continuous, (cf. Urysohn's Lemma. [5], 4.15.). Therefore

$$
L(\tilde{f})=\int_{a}^{b} \tilde{f} d \mu=\int_{K} d \mu+\int_{K^{c} \backslash F_{\varepsilon}} \tilde{f} d \mu+\int_{F_{\varepsilon}} \tilde{f} d \mu
$$

The third integral on the right is equal zero, by definition of $\tilde{f}$. We can estimate the second integral as follows,

$$
0 \leq \int_{K^{c} \backslash F_{e}} \tilde{f} d \mu \leq \int_{K^{c} \backslash F_{\varepsilon}} d \mu=\mu\left(K^{c} \backslash F_{\varepsilon}\right)<\varepsilon
$$

since $\tilde{f} \leq 1$ and using (3.3). Then

$$
L(\tilde{f})<\int_{K} d \mu+\varepsilon=\mu(K)+\varepsilon .
$$

We have that

$$
\mu(\mathrm{K})+\mu\left(\mathrm{K}^{\mathrm{c}}\right)=\int_{\mathrm{a}}^{\mathrm{b}} \mathrm{d} \mu=\mathrm{L}\left(\mathbf{1}_{[\mathrm{a}, \mathrm{b}]}\right)=\mathrm{L}(\tilde{\mathrm{f}})<\mu(\mathrm{K})+\varepsilon,
$$

The third equality follows from $r(\tilde{f})=r\left(\mathbf{1}_{[a, b]}\right)$. Thus $0 \leq \mu\left(K^{c}\right)<\varepsilon$, since $\mu(K)<\infty$.

To conclude, let $f \in \mathcal{C}(K)$ and $f^{*}$ a continuous extension of $f$ to the closed interval $[a, b]$. We can do this extension taking, for example, straight lines as follows: since $\mathrm{K}^{\mathrm{c}}$ is an open subset of $[a, b]$, it is at most a countable union of pairwise disjoint open intervals $\left(\alpha_{i}, \beta_{i}\right)$ 
intersected with the interval $[a, b]$, (see Lindeloef's thm., [18] Prop.9. p.40). For $x \in\left(\alpha_{i}, \beta_{i}\right)$ we define

$$
f^{*}(x)=(1-t) f\left(\alpha_{i}\right)+t f\left(\beta_{i}\right)
$$

if $x=\alpha_{i}(1-t)+t \beta_{i}$ for $t \in(0,1)$. The function $f^{*}$ is continuous on the interval $[a, b]$ since on $\mathrm{K}$ coincides with the continuous function $\mathrm{f}$ and on $\mathrm{K}^{\mathrm{c}}$ consists of straight lines, (cf. Tietze's Theorem [5], 4.16).

Then we have

$$
\ell(f)=\ell\left(r\left(f^{*}\right)\right)=L f^{*}=\int_{a}^{b} f^{*} d \alpha=\int_{a}^{b} f^{*} d \mu=\int_{K} f^{*} d \mu=\int_{K} f d \mu .
$$

as was to be shown.

ii) Conservation of norm. Take $f \in \mathcal{C}(K)$ such that $\|f\|_{\infty} \leq 1$. Since (3.1) holds we have,

$$
|\ell(f)|=\left|\int_{K} f d \mu\right| \leq\|f\|_{\infty} \mu(K) \leq \mu(K) .
$$

For the reverse inequality, let $\mathbf{1}(x)=1$ for all $x$, as defined in remark (3), so

$$
\|\ell\| \geq|\ell(1)|=\left|\int_{K} 1 \mathrm{~d} \mu\right|=\mu(\mathrm{K})
$$

we can conclude that $\mu(\mathrm{K})=\|\ell\|$.

iii) Uniqueness. Suppose $\mu$ and $v$ are finite measures that satisfy (3.1). Since $\mu$ and $v$ are regular measures, from (a) in the Appendix, it is enough to show that $\mu(C)=v(C)$ for any closed set $C$ of $K$. Let $C$ a nonempty closed set of $K$ and set $f_{k}(x):=\max \{0,1-k d(x, C)\}$ for all $k$ and $x \in K$, where $d(x, C)=\inf _{y \in C}|x-y|$. These functions are bounded, by 0 and 1 , and continuous. Thus $f_{k}$ belongs to $\mathcal{C}(K)$ for all $k$. Notice that they form a sequence that decreases to the indicator of $C$, i.e., $f_{k} \downarrow \mathbf{1}_{C}$, where $\mathbf{1}_{C}(x)=1$ if $x \in C$ and $\mathbf{1}_{C}(x)=0$ if $x \notin C$. Thus, for all $k$ we must have that $\int_{K} f_{k} d \mu=\int_{K} f_{k} d v$, and so we can use the dominated convergence theorem, see (b) in the Appendix, to conclude that

$$
\mu(C)=\lim _{k} \int_{K} f_{k} d \mu=\lim _{k} \int_{K} f_{k} d v=v(C) .
$$

\section{Remarks}

(a) It is possible to represent the linear positive functionals acting on $\mathcal{C}(\mathrm{K})$ as Riemann-Stieltjes integrals, similar to the original work of F. Riesz. This follows immediately from the chain of 
equalities (3.4). The caveat is that we cannot use $f$ directly in order to define the RiemannStieltjes integral, but any continuous extension of $f$ works, cf. theorem 3.2 below. This integral is independent of the extension of $f$.

(b) As just seen, the use of compact set $\mathrm{K}$ above allows us to extend the continuous functions to the entire interval $[a, b]$, using an elementary version of the Tietze's theorem. This construction is in general not possible if $\mathrm{K}$ is an arbitrary subset of the real line.

\section{$3.1 \quad$ Isomorphic spaces}

As a consequence of the previous results, we shall see that two spaces of functionals are practically the same. One of the spaces consists of Lebesgue integrals on compact subsets of $[a, b]$ and the other of Riemann-Stieltjes integrals over the whole interval $[a, b]$. In this way we show how the Lebesgue integral representation, that was introduced to represent functionals in the case of general compact sets, can be seen as a Riemann-Stieltjes integral. To state this precisely we need to introduce the terms isomorphic and constant in $\mathrm{K}^{\mathrm{c}}$.

A transformation $T$ which preserves the norm, that is $\|\mathrm{T} x\|=\|x\|$, is called an isometry. Two normed vector spaces $X$ and $Y$ are said to be isomorphic if there is a linear, bijective, isometry $\mathrm{T}: \mathrm{X} \rightarrow \mathrm{Y}$. Such functions are called isomorphisms. Since an isomorphism preserves the linear as well as the metric structure of the spaces, two isomorphic spaces can be considered identical, the isomorphism corresponding just to a labeling of the elements. We say that the monotone function $\alpha$ is constant in $\mathrm{K}^{\mathrm{c}}$ if it is constant in each interval of $\mathrm{K}^{\mathrm{c}}$.

Recall that $\mathcal{C}(\mathrm{X})_{+}^{*}$ denotes the set of positive linear functionals on $\mathcal{C}(\mathrm{X})$. Let $\mathrm{L}_{\alpha}$ denote the functional with corresponding monotone function $\alpha$ as introduced in (2.1).

The result mentioned above can be then stated as follows:

Theorem 3.2. The normed spaces $\left\{\mathrm{L}_{\alpha} \in \mathcal{C}[\mathrm{a}, \mathrm{b}]_{+}^{*}: \alpha\right.$ is constant in $\left.\mathrm{K}^{\mathrm{c}}\right\}$ and $\mathcal{C}(\mathrm{K})_{+}^{*}$ are isomorphic.

Before we prove this theorem we need two preparatory results.

Proposition 3.3. $r^{\mathrm{t}}: \mathcal{C}(\mathrm{K})_{+}^{*} \rightarrow \mathcal{C}[\mathrm{a}, \mathrm{b}]_{+}^{*}$ is an isometry.

Proof. $\left\|\mathrm{r}^{\mathrm{t}} \ell\right\|_{\mathcal{C}[\mathrm{a}, \mathrm{b}]_{+}^{*}}=\mathrm{V}(\alpha)=\alpha(\mathrm{b})-\alpha(\mathrm{a})=\mu([\mathrm{a}, \mathrm{b}])=\mu(\mathrm{K})+\mu([\mathrm{a}, \mathrm{b}] \backslash \mathrm{K})=\mu(\mathrm{K})=\|\ell\|_{\mathcal{C}(\mathrm{K})_{+}^{*}}$.

The first equality follows from Theorem 2.1. The function $\alpha$ depends on $\ell$. The second is the definition of the total variation of $\alpha$ and the third is the definition of $\mu$. The last two equalities follow from the construction of Theorem 3.1 . 
We denote the range of $r^{t}$ by Rang $r^{t}=\left\{L \in \mathcal{C}[a, b]_{+}^{*}: \exists l \in \mathcal{C}(K)_{+}^{*}\right.$ s.t. $\left.L=r^{t} l\right\}$

\section{Proposition 3.4.}

$$
\text { Rang } \mathrm{r}^{\mathrm{t}}=\left\{\mathrm{L}_{\alpha} \in \mathcal{C}[\mathrm{a}, \mathrm{b}]_{+}^{*}: \alpha \text { is constant in } \mathrm{K}^{\mathrm{c}}\right\}
$$

Proof. "C"

Let $\mathrm{L} \in \operatorname{Rang} \mathrm{r}^{\mathrm{t}} \subset \mathcal{C}[\mathrm{a}, \mathrm{b}]_{+}^{*}$. Then there exists $\ell \in \mathcal{C}(\mathrm{K})_{+}^{*}$ such that as in (3.2)

$$
r^{t}(\ell)(f)=L f=L_{\alpha} f=\int_{a}^{b} f d \alpha=\int_{a}^{b} f d \mu
$$

As was shown in the proof of Theorem 3.1 i), $\mu\left(K^{c}\right)=0$. Since $K^{c}$ is a countable union of intervals, these have $\mu$ measure zero. By the relation which is given in (4.1) below, between the measure $\mu$ and the monotone function $\alpha$ we conclude that $\alpha$ is constant in each one of the intervals of $K^{c}$.

$" \supset "$

Let $\mathrm{L}_{\alpha} \in \mathcal{C}[\mathrm{a}, \mathrm{b}]_{+}^{*}$ with $\alpha$ constant in each interval of $\mathrm{K}^{\mathrm{c}}$ and $\mu$ be the measure associated with this $\alpha$, as in Appendix (c). Define $\ell \in \mathcal{C}(K)_{+}^{*}$ as $\ell h=\int_{K} h d \mu$. We shall show that $r^{t}(\ell)(f)=L_{\alpha} f$ for every $f \in \mathcal{C}[a, b]$. Since $\alpha$ constant in each interval of $K^{c}$ this implies, using again (4.1), that $\mu\left(K^{c}\right)=0$. Then we have

$$
\mathrm{L}_{\alpha} f=\int_{a}^{b} f d \alpha=\int_{a}^{b} f d \mu=\int_{K} f d \mu=\int_{K} r(f) d \mu=\ell(r(f))=r^{t}(\ell)(f)
$$

where $r(f)$ denotes, as in Theorem (3.1) i) above, the restriction of $f$ to $K$.

Proof of Theorem 3.2.

From Proposition 3.3 and Proposition 3.4 it follows that $r^{t}$ is a bijective isometry. Since $r^{t}$ is linear as follows from its definition, then it is an isomorphism.

Acknowledgments We thank C. Bosch and Ma. C. Arrillaga for useful comments. We are grateful to Ma. R. Sanchez for her help in the search of bibliographical information.

\section{Appendix}

A collection of subsets $\mathcal{A}$ of $\mathrm{X}$ is called an $\sigma$-algebra if it is closed under finite (countable) union, complements and $\mathrm{X} \in \mathcal{A}$. If our space is $\mathbb{R}$, the Borel $\sigma$-algebra, $\mathcal{B}_{\mathbb{R}}$, is the smallest $\sigma$-algebra containing all the open intervals. A function $\mu: \mathcal{A} \rightarrow[0, \infty]$, where $\mathcal{A}$ is a $\sigma$-algebra, it is called a measure if it is countable additive, that is $\mu\left(\bigcup A_{n}\right)=\sum \mu\left(A_{n}\right)$ whenever $\left\{A_{n}\right\}$ is a disjoint sequence of elements in $\mathcal{A}$, and $\mu(\varnothing)=0$. A Borel measure is a measure defined on $\mathcal{B}_{\mathbb{R}}$. We say 
that a measure is regular if every measurable set can be approximated from above by open measurable sets and from below by compact measurable sets. A function $f$ from $(X, \mathcal{A}, \mu)$ to $\left(\mathbb{R}, B_{\mathbb{R}}\right)$ is $\mathcal{A}$-measurable if $\{x: \mathrm{f}(\mathrm{x}) \leq \mathrm{t}\} \in \mathcal{A}$ for all $\mathrm{t} \in \mathbb{R}$.

The following results are used in the proof of Theorem 3.1 .

(a) Every Borel measure in a metric space is regular. We will only use inner regularity, that is, for every Borel set $A$ and every $\varepsilon>0$ there exist a compact set $F_{\varepsilon}$ such that $F_{\varepsilon} \subset A$ and $\mu\left(A \backslash F_{\epsilon}\right)<\epsilon .2$ Thm 7.1.7. or [3] Lemma 1.5.7.

(b) (Dominated convergence theorem) Let $(X, \mathcal{A}, \mu)$ a measure spaces. Let $\mathrm{g}$ be a $[0, \infty]$-valued integrable function on $X$, that is, $\int g d \mu<\infty$, and let $f, f_{1}, f_{2}, \ldots$ real-valued $\mathcal{A}$-measurable functions on $X$ such that $f(x)=\lim _{n} f_{n}(x)$ and $\left|f_{n}(x)\right| \leq g(x)$. Then $f$ and $\left\{f_{n}\right\}$ are integrable and $\int f d \mu=\lim _{n} \int f_{n} d \mu$.

(c) Given a normalized monotone function $\alpha$ in the closed interval $[a, b]$, there is a unique Borel measure $\mu$ associated with it. This can be seen as follows (see for example [4): for $\mathrm{a} \leq \mathrm{s} \leq \mathrm{t} \leq \mathrm{b}$ let define $\langle\mathrm{s}, \mathrm{t}]$ where

Let

$$
\mathcal{F}_{0}=\left\{\bigcup_{\text {finite }}\left\langle s_{k}, t_{k}\right]:\left\langle s_{k}, t_{k}\right] \subset[a, b] \text { pairwise disjoint }\right\}
$$

Then $\mathcal{F}_{0}$ is an algebra of subsets of $[a, b]$ and therefore we can define a set function as

$$
\mu_{0}\left(\bigcup_{\text {finite }}\left\langle s_{k}, t_{k}\right]\right)=\sum_{\text {finite }} \alpha\left(t_{k}\right)-\alpha\left(s_{k}\right)
$$

Moreover, $\mu_{0}$ has a unique extension to a measure in the smallest $\sigma$-algebra containing $\mathcal{F}_{0}$ (Caratheodory's Theorem). See [1] . Moreover, for any continuous function $f$ it happens that

$$
\int_{a}^{b} f d \alpha=\int_{a}^{b} f d \mu
$$

where the integral on the left is a Riemann-Stieltjes integral, whereas the integral on the right is an integral in the sense of Lebesgue.

\section{References}

[1] Bartle, Robert G. The elements of integration. John Wiley \& Sons, Inc., New York-LondonSydney $1966 \mathrm{x}+129 \mathrm{pp}$.

[2] V. I. Bogachev, Measure theory. Vol. I, II, Springer-Verlag, Berlin, 2007. 
[3] D. Cohn, Measure theory, secon ed., Birkhäuser, Boston, Mass. 2013.

[4] Doob, J. L. Measure theory. Graduate Texts in Mathematics, 143. Springer-Verlag, New York, 1994. xii+210 pp. ISBN: 0-387-94055-3

[5] Gerald B. Folland, Real analysis, second ed., Pure and Applied Mathematics (New York), John Wiley \& Sons, Inc., New York, 1999, Modern techniques and their applications, A Wiley-Interscience Publication.

[6] Gray, J. D. The shaping of the Riesz representation theorem: a chapter in the history of analysis. Arch. Hist. Exact Sci. 31 (1984), no. 2, 127-187.

[7] D. G. Hartig, The Riesz Representation Theorem Revisited, Amer. Math. Monthly 90 No. 4 (1983), pp. 277-280

[8] E. Helly, Über lineare Funktionaloperationen, Wien Ber. 121 (1912), 265-297.

[9] Shizuo Kakutani, Concrete representation of abstract (M)-spaces. (A characterization of the space of continuous functions.), Ann. of Math. (2) 42 (1941), 994-1024.

[10] Erwin Kreyszig, Introductory functional analysis with applications, John Wiley \& Sons, New York-London-Sydney, 1978.

[11] A. Markoff, On mean values and exterior densities, Mat. Sbornik 4 (46) (1938), no. 1, 165-191.

[12] Johann Radon, Gesammelte Abhandlungen. Band 1, Verlag der Österreichischen Akademie der Wissenschaften, Vienna; Birkhäuser Verlag, Basel, 1987, With a foreword by Otto Hittmair, Edited and with a preface by Peter Manfred Gruber, Edmund Hlawka, Wilfried Nöbauer and Leopold Schmetterer.

[13] Michael Reed and Barry Simon, Methods of modern mathematical physics. I, second ed., Academic Press Inc. [Harcourt Brace Jovanovich Publishers], New York, 1980, Functional analysis.

[14] F. Riesz, Sur les opérations fonctionnelles linéares, Comptes Rendus Acad. Sci. Paris 149 (1909), 974-977.

[15] Demonstration nouvelle d'un théorème concernant les opérations, Annales Ecole Norm. Sup. 31 (1914), 9-14.

[16] Frédéric Riesz, Sur la représentation des opérations fonctionnelles linéaires par des intégrales de Stieltjes, Comm. Sém. Math. Univ. Lund [Medd. Lunds Univ. Mat. Sem.] 1952 (1952), no. Tome Supplementaire, 181-185.

[17] Frigyes Riesz and Béla Sz.-Nagy, Functional analysis, Dover Books on Advanced Mathematics, Dover Publications, Inc., New York, 1990, Translated from the second French edition by Leo F. Boron, Reprint of the 1955 original. 
[18] H. L. Royden, Real analysis, The Macmillan Co., New York; Collier-Macmillan Ltd., London, 1963.

[19] Walter Rudin, Real and Complex Analysis 3rd. ed., McGraw-Hill, Inc., 1987, New York, NY, USA.

[20] S. Saks, Integration in abstract metric spaces, Duke Math. J. 4 (1938), no. 2, 408-411.

[21] Stanisław Saks, Theory of the integral, Second revised edition. English translation by L. C. Young. With two additional notes by Stefan Banach, Dover Publications, Inc., New York, 1964.

[22] Martin Schechter, Principles of functional analysis, second ed., Graduate Studies in Mathematics, vol. 36, American Mathematical Society, Providence, RI, 2002.

[23] Barry Simon, Real analysis, A Comprehensive Course in Analysis, Part 1, American Mathematical Society, Providence, RI, 2015, With a 68 page companion booklet. 